\title{
Decentralized Variable Gain Robust Controllers with Guaranteed L2 Gain Performance for a Class of Uncertain Large-Scale Interconnected Systems with State Delays
}

\author{
Shunya NAGAI ${ }^{a}$, Hidetoshi OYA and Tomohiro KUBO ${ }^{a}$ \\ Tokushima University, 2-1 Minamijosanjima, Tokushima, 770-8506, JAPAN
}

\begin{abstract}
In this paper, we propose a decentralized variable gain robust controller with guaranteed $L_{2}$ gain performance for a class of uncertain large-scale interconnected systems with state delays. The proposed decentralized robust controller consists of a fixed gain and a variable gain tuned by parameter adjustment laws. In this paper, it is shown that sufficient conditions for the existence of the proposed decentralized variable gain robust control system are given in terms of LMIs. Finally, a simple illustrative example is shown.
\end{abstract}

\section{Introduction}

In order to design control systems, the derivation of a mathematical model for the controlled system is needed. However, there inevitably exist some gaps between the controlled system and its mathematical model. Therefore, robust control for uncertain dynamical systems has been widely studied and a great many results have been obtained on the problems of robust stability analysis and robust stabilization (e.g. [1] and references therein). Moreover, several variable gain robust state feedback controllers for uncertain systems have also been proposed. (e.g. [2], [3]). In the work of Oya and Hagino[2], a robust controllers with adaptive compensation inputs which achieve not only robust stability but also satisfactory transient response has been proposed. Additionally, a robust controller with adaptation mechanism has been suggested and the robust controller is tuned on-line based on the information about parameter uncertainties[3].

On the other hand, due to the rapid development of industry in recent years, controlled systems become more complex and such complex systems should be considered as large-scale interconnected systems. Thus decentralized robust control of uncertain large-scale interconnected systems has also attracted the attention of many researchers (e.g [4]-[6]). In Mao and Lin [6] for largescale interconnected systems with unmodelled interactions, the aggregative derivations are tracked by using a model following technique with on-line improvement, and a sufficient condition for which the overall system when controlled by the completely decentralized control is asymptotically stable has been established. Furthermore, Nagai and Oya [7] have suggested a decentralized variable gain robust controller which achieves not only robust stability but also satisfactory transient behavior for a class of uncertain large-scale interconnected systems with state delays. Additionally, a decentralized variable gain robust controller with guaranteed L2 gain performace for a class of uncertain large-scale interconnected systems has also been proposed[8].

In this paper, on the basis of existing results[7], [8], we propose a decentralized variable gain robust controller with guaranteed L2 gain performance for a class of uncertain large-scale interconnected systems with state delays. For the uncertain large-scale interconnected system with state delays, uncertainties and interactions with consideration satisfy the matching condition. The proposed decentralized robust controllers are composed of a state feedback with a fixed gain matrix and a variable one determined by parameter adjustment law. In addition, LMI-based sufficient conditions for the existence of the proposed decentralized variable gain robust controller are derived.

This paper is organized as follows. Notations and useful lemmas which are used in this paper are shown in Section 2, and in Section 3, the class of uncertain largescale interconnected systems with state delays which are considered in this paper is introduced. The main results are presented in Section 4, i.e. LMI-based sufficient conditions for the existence of the proposed decentralized variable gain robust controller are presented. Finally, a simple illustrative example is included.

\section{Notation and Lemmas}

In this section, we introduce notations, and useful and well-known lemmas (see [9], [10] for details) which are used in this paper as well as the existing work [11].

\footnotetext{
${ }^{a}$ Corresponding author: s-nagai@ee.tokushima-u.ac.jp
} 
In the paper, the following notations are used. For a matrix $X$, the inverse of the matrix $X$ and the transpose of one are denoted by $X^{-1}$ and $X^{T}$, respectively. Additionally $H_{e}\{X\}$ and $I_{n}$ mean $X+X^{T}$ and $n$-dimensional identity matrix, respectively, and the notation $\operatorname{diag}\left(X_{1}, \ldots, X_{M}\right)$ represents a block diagonal matrix composed of matrices $X_{i}$ for $i=1, \ldots, M$. For real symmetric matrices $X$ and $Y, X>Y$ (resp. $X \geq Y$ ) means that $X-Y$ is positive (resp. nonnegative) definite matrix. For a vector $\alpha \in \mathfrak{R}^{n},\|\alpha\|$ denotes the standard Euclidian norm, and for a matrix $X,\|X\|$ represents its induced norm. The symbol “* " means symmetric blocks in matrix inequalities.

Lemma 1: For arbitrary vectors $\alpha$ and $\beta$ and the matrices $X$ and $Y$ which have appropriate dimensions, the following inequality holds.

$$
2 \alpha^{T} X \Delta(t) Y \beta \leq 2\left\|X^{T} \alpha\right\|\|Y \beta\|
$$

where $\Delta(t) \in \mathfrak{R}^{s \times t}$ is a time-varying matrix satisfying the relation $\|\Delta(t)\| \leq 1.0$.

Lemma 2: (Schur complement) For a given constant real symmetric matrix $\Theta$, the following items are equivalent.

$$
\text { (i) } \Theta=\left(\begin{array}{ll}
\Theta_{11} & \Theta_{12} \\
\Theta_{12}^{T} & \Theta_{22}
\end{array}\right)>0 \text {, }
$$

(ii) $\Theta_{11}>0$ and $\hat{\Theta}_{22} \equiv \Theta_{22}-\Theta_{12}^{T} \Theta_{11}^{-1} \Theta_{12}>0$,

(iii) $\Theta_{22}>0$ and $\hat{\Theta}_{11} \equiv \Theta_{11}-\Theta_{12} \Theta_{22}^{-1} \Theta_{12}^{T}>0$.

\section{Problem Formulation}

Consider the uncertain large-scale interconnected system with state delays composed of $N$ subsystems described as (1)

$$
\begin{aligned}
\frac{d}{d t} x_{i}(t)= & A_{i i}(t) x_{i}(t)+\sum_{\substack{j=1 \\
j \neq i}}^{N} A_{i j}(t) x_{j}(t) \\
& +\sum_{j=1}^{N} H_{i j}(t) x_{j}\left(t-h_{i j}\right)+B_{i} u_{i}(t)+\Gamma_{x_{i}} \omega_{i}(t), \\
z_{i}(t)= & C_{i} x_{i}(t)+\Gamma_{z_{i}} \omega_{i}(t) .
\end{aligned}
$$

In $(1), x_{i}(t) \in \mathfrak{R}^{n_{i}}, u_{i}(t) \in \mathfrak{R}^{m_{i}}, z_{i}(t) \in \mathfrak{R}^{p_{i}}$ and $\omega_{i}(t) \in \mathfrak{R}^{q_{i}}$ are the vectors of the state, the control input, the controlled output and the disturbance input for the $i$-th subsystem, respectively. Besides,

$$
\begin{aligned}
& x(t)=\left(x_{1}^{T}(t), \ldots, x_{\mathrm{N}}^{T}(t)\right)^{T}, u(t)=\left(u_{1}^{T}(t), \ldots, u_{\mathrm{N}}^{T}(t)\right)^{T}, \\
& z(t)=\left(z_{1}^{T}(t), \ldots, z_{N}^{T}(t)\right)^{T} \\
& \omega(t)=\left(\omega_{1}^{T}(t), \ldots, \omega_{\mathrm{N}}^{T}(t)\right)^{T} \text { and }
\end{aligned}
$$
input, the controlled output and the disturbance input of the overall system. The matrices $A_{i i}(t), A_{i j}(t)$ and $H_{i j}(t)$ are given by

$$
\begin{aligned}
& A_{i i}(t)=A_{i i}+B_{i} \Delta_{i i}(t) L_{i}, \\
& A_{i j}(t)=B_{i} D_{i j}+B_{i} \Delta_{i j}(t) M_{i j}, \\
& H_{i j}(t)=B_{i} E_{i j}+B_{i} \Delta_{h_{i j}}(t) N_{h_{i j}},
\end{aligned}
$$

i.e. the uncertainties, the interactions, and coefficients of state delays satisfy the matching condition. In (1) and (2), the matrices $A_{i i} \in \mathfrak{R}^{n_{i} \times n_{i}}, B_{i} \in \mathfrak{R}^{n_{i} \times m_{i}}, C_{i} \in \mathfrak{R}^{p_{i} \times n_{i}}$, $\Gamma_{x_{i}} \in \mathfrak{R}^{n_{i} \times q_{i}}$ and $\Gamma_{z_{i}} \in \mathfrak{R}^{p_{i} \times q_{i}}$ are known system parameters and the matrices $L_{i}, D_{i j}, M_{i j}, E_{i j}$ and $N_{h_{i j}}$ which have appropriate dimensions represent the structure of uncertainties, interactions and state delays. Additionally, matrices $\quad \Delta_{i i}(t) \in \mathfrak{R}^{m_{i} \times r_{i}}$, $\Delta_{i j}(t) \in \mathfrak{R}^{m_{i} \times s_{i j}}$ and $\Delta_{h i j}(t) \in \mathfrak{R}^{m_{i} \times t_{i j}}$ denote unknown parameters satisfying the relations $\left\|\Delta_{i i}(t)\right\| \leq 1.0$, $\left\|\Delta_{i j}(t)\right\| \leq 1.0$ and $\left\|\Delta_{h_{i j}}(t)\right\| \leq 1.0$ respectively.

Now for the $i$-th subsystem of (1), we define the following control input.

$$
u_{i}(t) \equiv F_{i} x_{i}(t)+G_{i}\left(x_{i}, t\right) x_{i}(t)
$$

In (3), $F_{i} \in \mathfrak{R}^{m_{i} \times n_{i}}$ and $G_{i} \in \mathfrak{R}^{m_{i} \times n_{i}}$ are the fixed compensation gain matrix and the variable one for the $i$. th subsystem of (1). From (1), (2) and (3), the following closed-loop subsystem can be obtained.

$$
\begin{aligned}
\frac{d}{d t} x_{i}(t)= & \left(A_{i i}+B_{i} F_{i}\right) x_{i}(t)+B_{i} \Delta_{i i}(t) L_{i} x_{i}(t) \\
& +B_{i} \sum_{\substack{j=1 \\
j \neq i}}^{N}\left(D_{i j}+\Delta_{i i}(t) M_{i j}\right) x_{j}(t) \\
& +B_{i} \sum_{j=1}^{N}\left(E_{i j}+\Delta_{i i}(t) N_{h_{i j}}\right) x_{j}\left(t-h_{i j}\right) \\
& +\Gamma_{x_{i}} \omega_{i}(t)+B_{i} G_{i}\left(x_{i}, t\right) x_{i}(t) .
\end{aligned}
$$

Now we will give the definition of the decentralized variable gain robust control with guaranteed L2 gain performance $\gamma^{*}>0$ [12]. 
Definition 1: For the uncertain large-scale interconnected system of (1), the control input of (3) is said to be a decentralized variable gain robust control with guaranteed L2 gain performance $\gamma^{*}>0$ if the resultant closed-loop system of (4) is internally stable, and $H_{\infty}$-norm of the transfer function from the disturbance input $\omega(t)=\left(\omega_{1}^{T}(t), \ldots, \omega_{\mathrm{N}}^{T}(t)\right)^{T}$ to the controlled output $z(t)=\left(z_{1}^{T}(t), \ldots, z_{N}^{T}(t)\right)^{T}$ is less than or equal to a positive constant $\gamma^{*}$.

By using symmetric positive definite matrices $P_{i} \in \mathfrak{R}^{n_{i} \times n_{i}}$ and $P_{i j} \in \mathfrak{R}^{n_{i} \times n_{i}}$, we consider

$$
V(x, t) \equiv \sum_{i=1}^{N} V_{i}\left(x_{i}, t\right)
$$

where $V_{i}\left(x_{i}, t\right)$ is defined as

$$
V_{i}\left(x_{i}, t\right) \equiv x_{i}^{T}(t) P_{i} x_{i}(t)+\sum_{j=1}^{N} \int_{t-h_{i j}}^{t} x_{j}^{T}(\theta) P_{i j} x_{j}(\theta) d \theta
$$

Additionally, we define the Hamiltonian

$$
H(x, t) \equiv \frac{d}{d t} V(x, t)+\sum_{i=1}^{N}\left\{z_{i}^{T}(t) z_{i}(t)-\left(\gamma_{i}^{*}\right)^{2} \omega_{i}^{T}(t) \omega_{i}(t)\right\}
$$

Then we have the following lemma for the decentralized variable gain robust control with guaranteed L2 gain performance $\gamma^{*}$ [8].

Lemma 3: Consider the uncertain closed-loop subsystem of (4) with the control input of (3). For the quadratic function $V(x, t)$ and the signals $z(t)$ and $\omega(t)$, if there exist symmetric positive definite matrices $P_{i} \in \mathfrak{R}^{n_{i} \times n_{i}}$ and $P_{i j} \in \mathfrak{R}^{n_{i} \times n_{i}}$ and positive scalars $\gamma^{*}$ which satisfy the inequality

$$
H(x, t)<0
$$

then control input of (3) is the decentralized variable gain robust control with guaranteed L2 gain performance $\gamma^{*}$.

Proof : By integrating both sides of the inequality of (8) from 0 to $\infty$ with $x_{i}(0)=0$, we obtain the following inequality.

$$
V(x, \infty)+\sum_{i=1}^{\mathrm{N}}\left\{\int_{0}^{\infty} z_{i}^{T}(t) z_{i}(t) d t-\left(\gamma_{i}^{*}\right)^{2} \int_{0}^{\infty} \omega_{i}^{T}(t) \omega_{i}(t) d t\right\}<0 .
$$

We see from the inequality of (9) that the overall closed-loop subsystem of (4) is robustly stable (internally stable). Namely, robust stability of the uncertain closedloop subsystem is guaranteed and $H_{\infty}$-norm of the transfer function from the disturbance input
$\omega(t)=\left(\omega_{1}^{T}(t), \ldots, \omega_{N}^{T}(t)\right)^{T}$ to the controlled output $z(t)=\left(z_{1}^{T}(t), \ldots, z_{N}^{T}(t)\right)^{T}$ is less than a positive constant $\gamma^{*}$, because the inequality of (9) means the following relation

$$
\|z(t)\|_{L_{2}}<\gamma^{*}\|\omega(t)\|_{L_{2}}
$$

where $\gamma^{*}$ are given by

$$
\gamma^{*}=\max _{i} \gamma_{i}^{*}
$$

Thus the proof of Lemma 3 is accomplished.

From the above discussion, our design objective in this paper is to determine the decentralized variable gain robust control input of (3) such that the overall system achieves not only internal stability but also guaranteed L2 gain performance $\gamma^{*}$. That is to derive the symmetric positive definite matrices $P_{i} \in \mathfrak{R}^{n_{i} \times n_{i}}$ and $P_{i j} \in \mathfrak{R}^{n_{i} \times n_{i}}$, positive scalars $\gamma^{*}$, the fixed compensation gain $F_{i} \in \mathfrak{R}^{m_{i} \times n_{i}}$ and the variable one $G_{i}\left(x_{i}, t\right) \in \mathfrak{R}^{m_{i} \times n_{i}}$ satisfying the inequality of (8) for all admissible uncertainties $\quad \Delta_{i i}(t) \in \mathfrak{R}^{m_{i} \times r_{i}} \quad, \quad \Delta_{i j}(t) \in \mathfrak{R}^{m_{i} \times s_{i j}} \quad$ and $\Delta_{h i j}(t) \in \mathfrak{R}^{m_{i} \times t_{i j}} \quad$ and the disturbance input $\omega(t) \in L_{2}[0, \infty)$.

\section{Decentralized Variable Gain Controllers}

The following theorem shows a sufficient condition for the existence of the proposed decentralized control system.

Theorem 1: Consider the uncertain subsystem of (1) and the control input of (3). If the LMIs

$$
\begin{gathered}
\left(\begin{array}{c:c:c}
H_{e}\left\{A_{i i} F_{i}+B_{i} W_{i}\right\} & \Gamma_{x_{i}}+Y_{i} C_{i}^{T} \Gamma_{z_{i}} & \Lambda_{i}\left(Y_{i}\right) \\
\hdashline * & \Gamma_{z_{i}}^{T} \Gamma_{z_{i}}-\gamma_{i} I_{q_{i}} & 0 \\
\hdashline * & * & -\Omega_{i}\left(Y_{j i}, \varepsilon_{i}\right)
\end{array}\right)<0 \\
\hdashline(12) \\
\hdashline\left(\begin{array}{c:cc}
-Y_{i j} & Y_{i j} E_{i j}^{T} & Y_{i j} N_{h_{i j}}^{T} \\
\hdashline * & -\delta_{i} I_{m_{i}} & 0 \\
\hdashline & * & -\delta_{i} I_{r_{i j}}
\end{array}\right)<0
\end{gathered}
$$

are feasible, by using symmetric positive definite matrices $Y_{i} \in \mathfrak{R}^{n_{i} \times n_{i}}$ and $Y_{i j} \in \mathfrak{R}^{n_{i} \times n_{i}}$, matrices $W_{i} \in \mathfrak{R}^{m_{i} \times n_{i}}$ and positive constants $\varepsilon_{i}$ and $\delta_{i}$ which satisfy the LMIs of (12) and (13), the fixed gain matrix 
$F_{i} \in \mathfrak{R}^{m_{i} \times n_{i}}$ and the variable one $G_{i}\left(x_{i}, t\right) \in \mathfrak{R}^{m_{i} \times n_{i}}$ are determined as $F_{i}=W_{i} Y_{i}^{-1}$ and

$G_{i}\left(x_{i}, t\right)=\left\{\begin{array}{l}-\frac{\left\|B_{i}^{T} P_{i} x_{i}(t)\right\| L_{i i} x_{i}(t)\left\|+\left\{\varepsilon_{i}(N-1)+\delta_{i} N\right\}\right\| B_{i}^{T} P_{i} x_{i}(t) \|^{2}}{\left\|B_{i}^{T} P_{i} x_{i}(t)\right\|^{2}} B_{i}^{T} P_{i} \\ G_{i}\left(x_{i}, t_{\varepsilon}\right)\end{array}\right.$ respectively. In (12) and (13), matrices $\Lambda_{i}\left(Y_{i}\right)$ and $\Omega_{i}\left(Y_{j i}, \varepsilon_{i}\right)$ are given by

$$
\begin{aligned}
& \Lambda_{i}\left(Y_{i}\right)=(\overbrace{Y_{i} \quad Y_{i} \quad Y_{i}}^{N} Y_{i} \quad Y_{i} C_{i}^{T} \quad Y_{i} D_{1 i}^{T} \quad Y_{i} M_{1 i}^{T} \quad \cdots \\
& \left.\begin{array}{lllllll}
Y_{i} D_{i-1 i}^{T} & Y_{i} M_{i-1 i}^{T} & Y_{i} D_{i+1 i}^{T} & Y_{i} M_{i+1 i}^{T} & \cdots & Y_{i} D_{N i}^{T} & Y_{i} M_{N i}^{T}
\end{array}\right) \text {, }
\end{aligned}
$$

$$
\begin{aligned}
& \Omega_{i}\left(Y_{j i}, \varepsilon_{i}\right)=\operatorname{diag}\left(Y_{1 i} \quad Y_{2 i} \quad \cdots \quad Y_{N i} \quad I_{p_{i}} \quad \varepsilon_{1} I_{m_{1}} \quad \varepsilon_{1} I_{s_{1 i}} \quad \cdots\right. \\
& \left.\cdots \varepsilon_{i-1} I_{m_{i-1}} \quad \varepsilon_{i-1} I_{s_{i-1 i}} \quad \varepsilon_{i+1} I_{m_{i+1}} \quad \varepsilon_{i+1} I_{s_{i+1 i}} \quad \cdots \quad \varepsilon_{N} I_{m_{N}} \quad \varepsilon_{N} I_{s_{N i}}\right) .
\end{aligned}
$$

Moreover, $t_{\varepsilon}$ in (14) is given by $t_{\varepsilon}=\lim _{\varepsilon>0, \varepsilon \rightarrow 0}(t-\varepsilon)$ [3].

Then the control input of (3) is the decentralized variable gain robust control with guaranteed L2 gain performance $\gamma^{*}$.
Proof: In order to prove theorem 1, let us consider the quadratic function of (5), the Hamiltonian $H_{i}\left(x_{i}, t\right)$ of (7) and the inequality of (8).

For the quadratic functions $V_{i}\left(x_{i}, t\right)$ of (6), its time derivative can be computed as

$$
\begin{aligned}
\frac{d}{d t} V_{i}\left(x_{i}, t\right) & =x_{i}^{T}(t)\left[H_{e}\left\{\left(A_{i i}+B_{i} F_{i}\right)^{T} P_{i}\right\}\right] x_{i}(t)+H_{e}\left\{x_{i}^{T}(t) P_{i} B_{i} \Delta_{i i}(t) L_{i} x_{i}(t)\right\}+H_{e}\left\{x_{i}^{T}(t) P_{i} B_{i} G_{i}\left(x_{i}, t\right) x_{i}(t)\right\} \\
& +H_{e}\left\{x_{i}^{T}(t) P_{i} B_{i} \sum_{j=1}^{N}\left(D_{i j}+\Delta_{i j}(t) M_{i j}\right) x_{j}(t)\right\}+H_{e}\left\{x_{i}^{T}(t) P_{i} B_{i} \sum_{j=1}^{N}\left(E_{i j}+\Delta_{h_{i j}}(t) N_{h_{i j}}\right) x_{j}\left(t-h_{i j}\right)\right\} \\
& +H_{e}\left\{x_{i}^{T}(t) P_{i} \Gamma_{x_{i}} \omega_{i}(t)\right\}+\sum_{j=1}^{N}\left(x_{j}^{T}(t) P_{i j} x_{j}(t)-x_{j}^{T}\left(t-h_{i j}\right) P_{i j} x_{j}\left(t-h_{i j}\right)\right) \\
\leq & x_{i}^{T}(t)\left[H_{e}\left\{\left(A_{i i}+B_{i} F_{i}\right)^{T} P_{i}\right\}\right] x_{i}(t)+2\left\|B_{i}^{T} P_{i} x_{i}(t)\right\| L_{i i} x_{i}(t) \| \\
& +2 x_{i}^{T}(t) P_{i} B_{i} G_{i}\left(x_{i}, t\right) x_{i}(t)+2 \varepsilon_{i}(N-1)\left\|B_{i}^{T} P_{i} x_{i}(t)\right\|\left\|^{2}+\delta_{i} N\right\| B_{i}^{T} P_{i} x_{i}(t) \|^{2} \\
& +\frac{1}{\varepsilon_{i}} \sum_{j=1}^{N} x_{j}^{T}(t)\left(D_{i j}^{T} D_{i j}+M_{i j}^{T} M_{i j}\right) x_{j}(t)+\frac{1}{\delta} \sum_{j=1}^{N} x_{j}^{T}\left(t-h_{i j}\right)\left(E_{i j}^{T} E_{i j}+N_{h_{i j}}^{T} N_{h_{i j}}\right) x_{j}\left(t-h_{i j}\right) \\
& +H_{e}\left\{x_{i}^{T}(t) P_{i} \Gamma_{x_{i}} \omega_{i}(t)\right\}+\sum_{j=1}^{N}\left(x_{j}^{T}(t) P_{i j} x_{j}(t)-x_{j}^{T}\left(t-h_{i j}\right) P_{i j} x_{j}\left(t-h_{i j}\right)\right)
\end{aligned}
$$

Note that for derivation of (17), Lemma 1 and the well-known inequality

$$
2 \alpha^{T} \beta \leq \delta \alpha^{T} \alpha+\frac{1}{\delta} \beta^{T} \beta
$$

for any vectors $\alpha$ and $\beta$ with appropriate dimensions and a positive scalar $\delta$ have been used.
Firstly, we consider the case of $B_{i}^{T} P_{i} x_{i}(t) \neq 0$. In this case, substituting the variable gain matrix of (14) into (17) and some algebraic manipulations give the following inequality. 
$\frac{d}{d t} V_{i}\left(x_{i}, t\right) \leq x_{i}^{T}(t)\left[H_{e}\left\{\left(A_{i i}+B_{i} F_{i}\right)^{T} P_{i}\right)\right] x_{i}(t)$

$$
\begin{aligned}
& +\frac{1}{\varepsilon_{i}} \sum_{\substack{j=1 \\
j \neq i}}^{N} x_{j}^{T}(t)\left(D_{i j}^{T} D_{i j}+M_{i j}^{T} M_{i j}\right) x_{j}(t) \\
& +\frac{1}{\delta_{i}} \sum_{\substack{j=1 \\
j \neq i}}^{N} x_{j}^{T}\left(t-h_{i j}\right)\left(E_{i j}^{T} E_{i j}+N_{h_{j}}^{T} N_{h_{j}}\right) x_{j}\left(t-h_{i j}\right) \\
& +H_{e}\left\{x_{i}^{T}(t) P_{i} \Gamma_{x_{i}} \omega_{i}(t)\right\} \\
& +\sum_{j=1}^{N}\left(x_{j}^{T}(t) P_{i j} x_{j}(t)-x_{j}^{T}\left(t-h_{i j}\right) P_{i j} x_{j}\left(t-h_{i j}\right)\right)
\end{aligned}
$$

holds, where $\gamma_{i} \equiv\left(\gamma_{i}^{*}\right)^{2}$. Hence from (5), (7), (19) and (20), the following relation for the Hamiltonian $H(x, t)$ can be derived.

$$
\begin{aligned}
H(x, t) \leq & \sum_{i=1}^{N} x_{i}^{T}(t)\left[H_{e}\left\{\left(A_{i i}+B_{i} F_{i}\right)^{T} P_{i}\right\}\right] x_{i}(t)+\sum_{i=1}^{N} \frac{1}{\varepsilon_{i}} \sum_{\substack{j=1 \\
j \neq i}}^{N} x_{j}^{T}(t)\left(D_{i j}^{T} D_{i j}+M_{i j}^{T} M_{i j}\right) x_{j}(t) \\
& +\sum_{i=1}^{N} \frac{1}{\delta_{i}} \sum_{j=1}^{N} x_{j}^{T}\left(t-h_{i j}\right)\left(E_{i j}^{T} E_{i j}+N_{h_{i j}}^{T} N_{h_{i j}}\right) x_{j}\left(t-h_{i j}\right)+\sum_{i=1}^{N} H_{e}\left\{x_{i}^{T}(t) P_{i} \Gamma_{x_{i}} \omega_{i}(t)\right\} \\
& +\sum_{i=1}^{N} \sum_{j=1}^{N} x_{j}^{T}(t) P_{i j} x_{j}(t)-\sum_{i=1}^{N} \sum_{j=1}^{N} x_{j}^{T}\left(t-h_{i j}\right) P_{i j} x_{j}\left(t-h_{i j}\right)+\sum_{i=1}^{N} x_{i}^{T}(t) C_{i}^{T} C_{i} x_{i}(t) \\
& +\sum_{i \sim 1}^{N} H_{e}\left\{x_{i}^{T}(t) C_{i}^{T} \Gamma_{z_{i}} \omega_{i}(t)\right\}+\sum_{i=1}^{N} \omega_{i}^{T}(t)\left(\Gamma_{z_{i}}^{T} \Gamma_{z_{i}}-\gamma_{i} I_{q_{i}}\right) \omega_{i}(t)
\end{aligned}
$$
(22).

In addition, the inequality of (21) can be rewritten as

$$
\begin{aligned}
H(x, t) \leq & \sum_{i=1}^{N} x_{i}^{T}(t)\left[H_{e}\left\{\left(A_{i i}+B_{i} F_{i}\right)^{T} P_{i}\right\}\right] x_{i}(t)+\sum_{i=1}^{N} \sum_{\substack{j=1 \\
j \neq i}}^{N} \frac{1}{\varepsilon_{j}} x_{i}^{T}(t)\left(D_{j i}^{T} D_{j i}+M_{j i}^{T} M_{j i}\right) x_{i}(t) \\
& +\sum_{i=1}^{N} \frac{1}{\delta_{i}} \sum_{j=1}^{N} x_{j}^{T}\left(t-h_{i j}\right)\left(E_{i j}^{T} E_{i j}+N_{h_{i j}}^{T} N_{h_{i j}}\right) x_{j}\left(t-h_{i j}\right)+\sum_{i=1}^{N} H_{e}\left\{x_{i}^{T}(t) P_{i} \Gamma_{x_{i}} \omega_{i}(t)\right\} \\
& +\sum_{i=1}^{N} x_{i}^{T}(t) \sum_{j=1}^{N}\left(P_{j i}\right) x_{i}(t)-\sum_{i=1}^{N} \sum_{j=1}^{N} x_{j}^{T}\left(t-h_{i j}\right) P_{i j} x_{j}\left(t-h_{i j}\right)+\sum_{i=1}^{N} x_{i}^{T}(t) C_{i}^{T} C_{i} x_{i}(t) \\
& +\sum_{i \sim 1}^{N} H_{e}\left\{x_{i}^{T}(t) C_{i}^{T} \Gamma_{z_{i}} \omega_{i}(t)\right\}+\sum_{i=1}^{N} \omega_{i}^{T}(t)\left(\Gamma_{z_{i}}^{T} \Gamma_{z_{i}}-\gamma_{i} I_{q_{i}}\right) \omega_{i}(t)
\end{aligned}
$$




$$
H(x, t) \leq \sum_{i=1}^{N}\left(\frac{x_{i}(t)}{-\frac{1}{\omega_{i}}(t)}\right)^{T} \Psi_{i}\left(P_{i}, P_{i j}, \varepsilon_{i}, \gamma_{i}\right)\left(\frac{x_{i}(t)}{\left.-\frac{1}{\omega_{i}(t)}\right)}\right)+\sum_{i=1}^{N} \frac{1}{\delta_{i}} \sum_{j=1}^{N} x_{j}^{T}\left(t-h_{i j}\right)\left(E_{i j}^{T} E_{i j}+N_{h_{i j}}^{T} N_{h_{i j}}-\delta_{i} P_{i j}\right) x_{j}\left(t-h_{i j}\right)
$$

where $\Psi_{i}\left(P_{i}, P_{i j} \varepsilon_{i}, \gamma_{i}\right)$ is given by

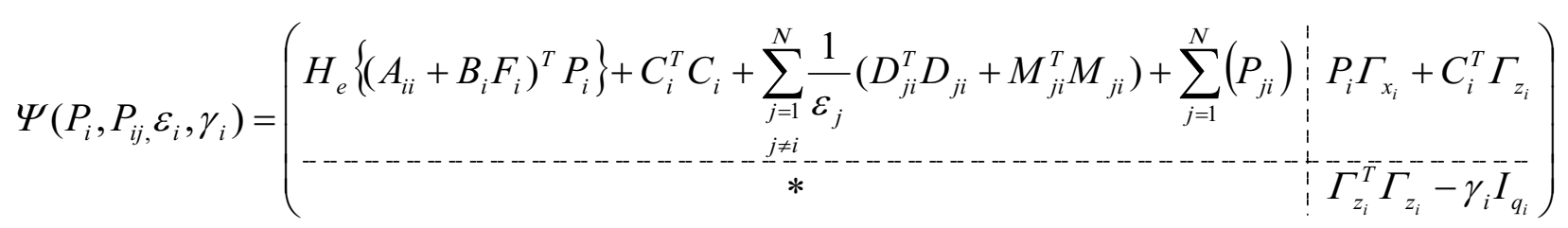

Therefore if the following matrix inequalities holds, then the relation of (8) for the Hamiltonian is satisfied.

$$
\Psi_{i}\left(P_{i}, P_{i j}, \varepsilon_{i}, \gamma_{i}\right)<0 \text { and }\left(E_{i j}^{T} E_{i j}+N_{h_{i j}}^{T} N_{h_{i j}}-\delta_{i} P_{i j}\right)<0 .
$$

Next we consider the case of $B_{i}^{T} P_{i} x_{i}(t)=0$. In this case, one can see from (17) and (20), the definition of the control input of (3) and the variable gain matrix of (14) that if matrix inequality of (25) holds, then the relation of (8) is also satisfied.
Finally, we consider the matrix inequalities of (25). By applying Lemma 2 (Schur complement) to the first matrix inequality of (25). we can obtain

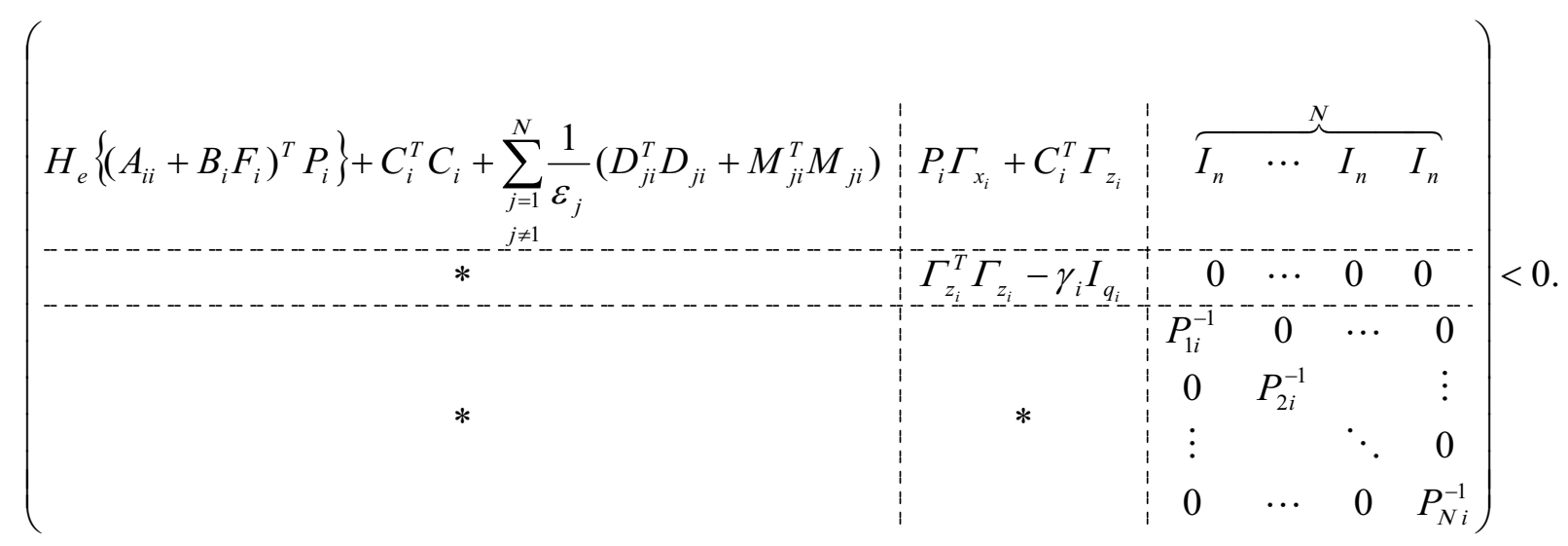

Furthermore by introducing the matrices $Y_{i} \equiv P_{i}^{-1}$, $Y_{i j} \equiv P_{i j}^{-1} \quad$ and $\quad W_{i} \equiv F_{i} Y_{i}$ and pre- and postmultiplying both sides of (26) and the second inequality of (25) by $\operatorname{diag}\left(Y_{i}, I_{q_{i}}, I_{n}, \cdots, I_{n}\right)$ and $Y_{i j}$ respectively, we have the following inequalities. 


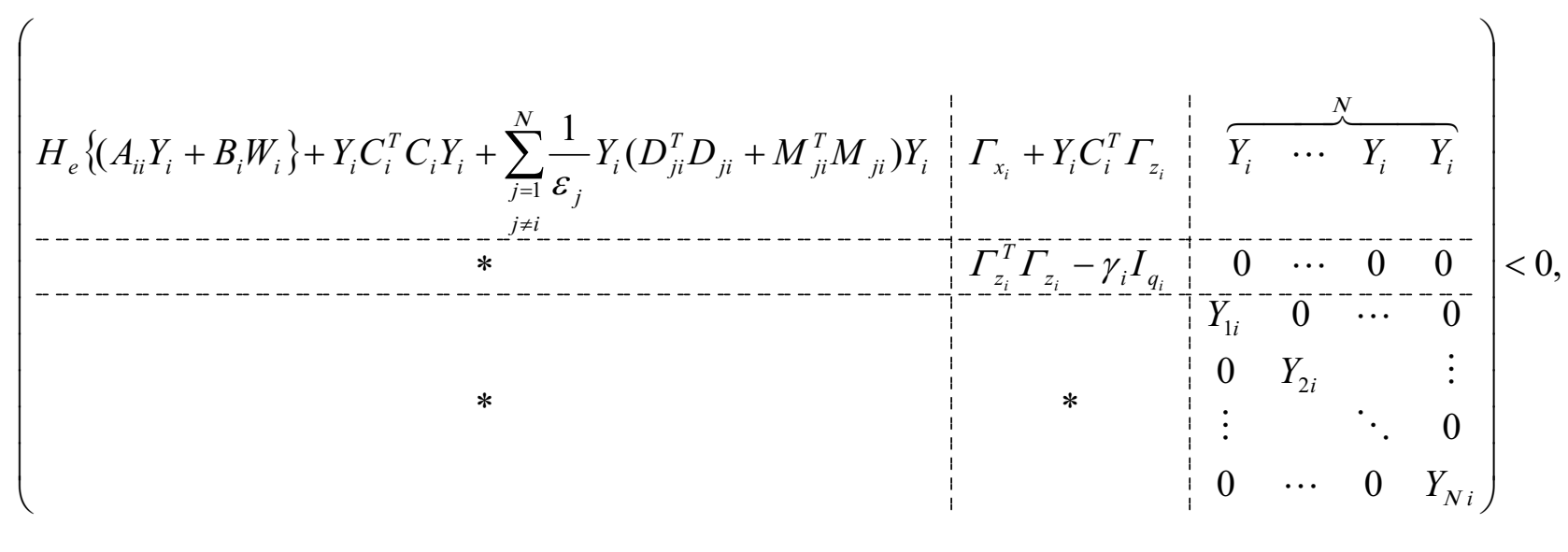

$$
Y_{i j}\left(E_{i j}^{T} E_{i j}+N_{h_{i j}}^{T} N_{h_{i j}}\right) Y_{i j}-\delta_{i} Y_{i j}<0
$$

Thus by applying Lemma 2 to (27) and (28), we find that these inequalities are equivalent to the LMIs of (12) and (13), respectively. Therefore by solving the LMIs of (12) and (13), the fixed gain matrix is determined as $F_{i} \equiv W_{i} Y_{i}^{-1}$, and the variable one is given by (14). Thus the proof of Theorem $\mathbf{1}$ is accomplished. dimensional subsystems, i.e. $N=3$. The system parameters under consideration are given as

\section{Numerical Examples}

In this example, we consider the uncertain large-scale interconnected system consisting of three two-

$$
\begin{aligned}
& A_{11}=\left(\begin{array}{cc}
-1.0 & 1.0 \\
0.0 & 1.0
\end{array}\right), A_{22}=\left(\begin{array}{cc}
0.0 & 1.0 \\
-1.0 & -1.0
\end{array}\right), A_{33}=\left(\begin{array}{cc}
1.0 & 0.0 \\
-1.0 & -3.0
\end{array}\right), B_{1}=\left(\begin{array}{l}
0.0 \\
1.0
\end{array}\right), B_{2}=\left(\begin{array}{l}
1.0 \\
1.0
\end{array}\right), B_{3}=\left(\begin{array}{l}
1.0 \\
1.0
\end{array}\right), \\
& L_{1}^{T}=\left(\begin{array}{l}
1.0 \\
0.0
\end{array}\right), L_{2}^{T}=\left(\begin{array}{l}
1.0 \\
2.0
\end{array}\right), L_{3}^{T}=\left(\begin{array}{l}
1.0 \\
1.0
\end{array}\right), D_{12}^{T}=\left(\begin{array}{l}
1.0 \\
2.0
\end{array}\right), D_{13}^{T}=\left(\begin{array}{l}
2.0 \\
1.0
\end{array}\right), D_{21}^{T}=\left(\begin{array}{l}
1.0 \\
0.0
\end{array}\right), D_{23}^{T}=\left(\begin{array}{l}
1.0 \\
1.0
\end{array}\right), D_{31}^{T}=\left(\begin{array}{l}
2.0 \\
1.0
\end{array}\right), \\
& D_{32}^{T}=\left(\begin{array}{l}
0.0 \\
2.0
\end{array}\right), M_{12}^{T}=\left(\begin{array}{l}
2.0 \\
1.0
\end{array}\right), M_{13}^{T}=\left(\begin{array}{l}
2.0 \\
2.0
\end{array}\right), M_{21}^{T}=\left(\begin{array}{l}
1.0 \\
0.0
\end{array}\right), M_{23}^{T}=\left(\begin{array}{l}
0.0 \\
3.0
\end{array}\right), M_{31}^{T}=\left(\begin{array}{l}
1.0 \\
2.0
\end{array}\right), M_{32}^{T}=\left(\begin{array}{l}
1.0 \\
1.0
\end{array}\right), \\
& E_{11}^{T}=\left(\begin{array}{l}
1.0 \\
2.0
\end{array}\right), E_{12}^{T}=\left(\begin{array}{l}
1.0 \\
1.0
\end{array}\right), E_{13}^{T}=\left(\begin{array}{l}
2.0 \\
1.0
\end{array}\right), E_{21}^{T}=\left(\begin{array}{l}
1.0 \\
1.0
\end{array}\right), E_{22}^{T}=\left(\begin{array}{l}
2.0 \\
1.0
\end{array}\right), E_{23}^{T}=\left(\begin{array}{l}
1.0 \\
0.0
\end{array}\right), E_{31}^{T}=\left(\begin{array}{l}
0.0 \\
2.0
\end{array}\right), E_{32}^{T}=\left(\begin{array}{l}
2.0 \\
2.0
\end{array}\right), \\
& E_{33}^{T}=\left(\begin{array}{l}
1.0 \\
0.0
\end{array}\right), N_{h_{11}}^{T}=\left(\begin{array}{l}
1.0 \\
1.0
\end{array}\right), N_{h_{12}}^{T}=\left(\begin{array}{l}
1.0 \\
1.0
\end{array}\right), N_{h_{13}}^{T}=\left(\begin{array}{l}
2.0 \\
3.0
\end{array}\right), N_{h_{21}}^{T}=\left(\begin{array}{l}
1.0 \\
1.0
\end{array}\right), N_{h_{22}}^{T}=\left(\begin{array}{l}
3.0 \\
1.0
\end{array}\right), N_{h_{23}}^{T}\left(\begin{array}{l}
0.0 \\
3.0
\end{array}\right), N_{h_{31}}^{T}=\left(\begin{array}{l}
1.0 \\
0.0
\end{array}\right), \\
& N_{h_{32}}^{T}=\left(\begin{array}{l}
3.0 \\
1.0
\end{array}\right), N_{h_{33}}^{T}=\left(\begin{array}{l}
2.0 \\
1.0
\end{array}\right), \Gamma_{x_{1}}=\left(\begin{array}{l}
1.0 \\
1.0
\end{array}\right), \Gamma_{x_{2}}=\left(\begin{array}{l}
1.0 \\
0.0
\end{array}\right), \Gamma_{x_{3}}=\left(\begin{array}{l}
1.0 \\
1.0
\end{array}\right), C_{1}^{T}=\left(\begin{array}{l}
1.0 \\
0.0
\end{array}\right), C_{2}^{T}=\left(\begin{array}{l}
1.0 \\
1.0
\end{array}\right), C_{3}^{T}=\left(\begin{array}{l}
1.0 \\
1.0
\end{array}\right), \\
& \Gamma_{z_{1}}=1.0, \Gamma_{z_{2}}=1.0, \Gamma_{z_{3}}=1.0 .
\end{aligned}
$$

Firstly, by using Theorem 1 we design the proposed decentralized variable gain robust controller. By solving LMIs of (12) and (13), we have 


$$
\begin{aligned}
& Y_{1}=\left(\begin{array}{cc}
1.0047 \times 10^{2} & 1.8667 \times 10^{1} \\
* & 1.3780 \times 10^{2}
\end{array}\right), Y_{2}=\left(\begin{array}{cc}
1.3464 \times 10^{2} & 7.8244 \times 10^{-3} \\
* & 1.3460 \times 10^{2}
\end{array}\right), \\
& Y_{3}=\left(\begin{array}{cc}
1.3441 \times 10^{2} & -4.4497 \times 10^{-2} \\
* & 1.3458 \times 10^{2}
\end{array}\right), W_{1}^{T}=\left(\begin{array}{c}
-1.3793 \\
-2.0519
\end{array}\right) \times 10^{2}, W_{2}^{T}=\left(\begin{array}{c}
-1.3793 \\
-2.0519
\end{array}\right) \times 10^{1}, \\
& W_{3}^{T}=\left(\begin{array}{cc}
-2.0418 \\
3.3353
\end{array}\right) \times 10^{2}, Y_{11}=\left(\begin{array}{cc}
1.0157 & -4.5676 \\
* & 5.5900
\end{array}\right) \times 10^{1}, Y_{12}=\left(\begin{array}{cc}
9.0619 & -4.3787 \\
* & 9.0621
\end{array}\right) \times 10^{1}, \\
& Y_{13}=\left(\begin{array}{cc}
6.5473 & -4.7474 \\
* & 5.3610
\end{array}\right) \times 10^{1}, Y_{21}=\left(\begin{array}{cc}
8.5075 & -4.9562 \\
* & 8.5075
\end{array}\right) \times 10^{1}, \\
& Y_{22}=\left(\begin{array}{cc}
2.5941 \times 10^{1} & -4.0509 \times 10^{1} \\
* & 1.1506 \times 10^{2}
\end{array}\right), Y_{23}=\left(\begin{array}{cc}
9.2729 & 0.0000 \\
* & 1.6562
\end{array}\right) \times 10^{1}, Y_{31}=\left(\begin{array}{cc}
9.1729 & 0.0000 \\
* & 3.6904
\end{array}\right) \times 10^{1}, \\
& Y_{32}=\left(\begin{array}{cc}
3.0817 & -3.6599 \\
* & 7.2644
\end{array}\right) \times 10^{1}, Y_{33}=\left(\begin{array}{cc}
4.0998 & -3.5477 \\
* & 1.1195
\end{array}\right) \times 10^{1} \\
& \varepsilon_{1}=7.8221, \varepsilon_{2}=4.5591, \quad \varepsilon_{3}=2.9293, \delta_{1}=2.7308 \times 10^{2}, \delta_{2}=2.3822 \times 10^{2}, \delta_{3}=2.5028 \times 10^{2}, \\
& \gamma_{1}=6.5765, \quad \gamma_{2}=3.2952, \quad \gamma_{3}=5.2439 .
\end{aligned}
$$

Thus the fixed gain matrices $F_{i} \in \mathfrak{R}^{1 \times 2}$ can be computed as

$$
F_{1}=(-1.1245-1.3367), \quad F_{2}=\left(\begin{array}{lll}
-5.0451 & 4.9566
\end{array}\right) \times 10^{1}, \quad F_{3}=\left(\begin{array}{lll}
-1.5183 & 2.4728
\end{array}\right)
$$

and the variable one $G_{i}\left(x_{i}, t\right) \in \mathfrak{R}^{1 \times 2}$ can also be derived. Furthermore, the positive scalars $\gamma_{i}^{*}=\sqrt{\gamma_{i}}$ can be obtaind as

$$
\gamma_{1}^{*}=2.5645, \quad \gamma_{2}^{*}=1.8153, \quad \gamma_{3}^{*}=2.2900 .
$$

Therefore, guaranteed L2 gain performance $\gamma^{*}=\max _{i} \gamma_{i}^{*}$ via the proposed controller is given by

$$
\gamma^{*}=2.5645
$$

Thus we can see that the proposed decentralized variable gain robust controller with guaranteed L2 gain performance can be obtained by solving LMIs of (12) and (13).

\section{Conclusions}

In this paper, for the uncertain large-scale interconnected system with state delays, we have proposed a decentralized variable gain robust controller which achieves not only robust stability but also guaranteed L2 gain performance.

In the future, we will extend the proposed controller to the design problem for such a broad class of systems as large-scale systems with mismatched uncertainties, largescale systems with Lipschitz nonlinearities and so on.

\section{References}

1. K. Zhou, Essentials of Robust Control, Prentice Hall Inc. (1998)

2. H. Oya and K. Hagino, Robust Control with Adaptive Compensation Input for Linear Uncertain Systems, IEICE Trans. Fundamentals of Electronics, Communications and Computer Sciences, vol.E86-A (6), pp.1517-1524 (2003)

3. M. Maki and K. Hagino, Robust Control with Adaptation Mechanism for Improving Transient Behavior, Int. J. Contr., 72(13), pp.1218-1226 (1999)

4. D. D. Sijjak, Decentralized Control of Complex Systems, Academic Press, New York (1991)

5. Y. H. Chen, Decentralized Robust Control System Design for Large-Scale Uncertain Systems, Int. J. Contr., 47(5), pp.1195-1205 (1988)

6. C. J. Mao. and W. S. Gong, Decentralized Control of Interconnected Systems with Unmodelled Nonlinearity and Interaction, Automatica, 26(2), pp.263-268 (1990)

7. S. Nagai and H. Oya, Decentralized Variable Gain Robust Controllers for a Class of Uncertain LargeScale Interconnected Systems with State Delays, Proc. of ADCONIP 2014 The 5th International Symposium on Advanced Control of Industrial Processes, No.1B1-3, pp.68-72, Hiroshima, JAPAN (2014) 
8. S. Nagai and H. Oya, Synthesis of Decentralized Variable Gain Robust Controllers with $\mathrm{L}_{2}$ Gain Performance for a Class of Uncertain Large-Scale Interconnected Systems, Journal of Control Science and Engineering, 8 pages (2015) (to appear)

9. F. R. Gantmacher, The Theory of Matrices, vol.1, Chelsea Publishing Company, New York (1960)

10. S. Boyd, L. El Ghaoui, E. Feron and V. Balakrishnan, Linear Matrix Inequalities in System and Control Theory, SIAM Studies in Applied Mathematics (1994)
11. H. Oya and K. Hagino, Synthesis of Adaptive Gain Robust Output Feedback Controllers for a Class of Lipschitz Nonlinear Systems with Unknown Upper Bound of Uncertainty, Journal of Control Science and Engineering, Vol.2012, No.Article ID 902576, pp.1-10, (2012)

12. H. Oya and K. Hagino, Robust Non-Fragile $H^{\infty}$ Controllers for Uncertain Linear Systems, Bulletin of the University of Electro-Communications, 18(12), pp.53-58, (2006) 